\title{
Impact of Liver Cirrhosis on Incidence of Dialysis Among Patients with Type 2 Diabetes
}

\author{
Yi-Jing Sheen • Pei-Tseng Kung • Wayne H.-H. Sheu • Wei-Yin Kuo • \\ Wen-Chen Tsai (iD
}

Received: June 14, 2020 / Published online: September 8, 2020

(C) The Author(s) 2020

\begin{abstract}
Introduction: Renal injury is a common complication of liver cirrhosis and type 2 diabetes mellitus (T2DM). The aim of this study was to analyze the association between cirrhosis and dialysis in patients with T2DM.

Methods: This was a retrospective study of specific patient populations using data collected from the
\end{abstract}

Pei-Tseng Kung and Wen-Chen Tsai contributed equally to this work.

Digital Features To view digital features for this article go to https://doi.org/10.6084/m9.figshare.12866273.

Electronic Supplementary Material The online version of this article (https://doi.org/10.1007/s13300020-00919-6) contains supplementary material, which is available to authorized users.

Y.-J. Sheen · W.-Y. Kuo · W.-C. Tsai ( $\varangle)$

Department of Health Services Administration, China Medical University, Taichung, Taiwan

e-mail: wctsai2011@gmail.com

Y.-J. Sheen · W. H.-H. Sheu

Division of Endocrinology and Metabolism, Department of Internal Medicine, Taichung

Veterans General Hospital, Taichung, Taiwan

Y.-J. Sheen

Department of Public Health, China Medical

University, Taichung, Taiwan

P.-T. Kung

Department of Healthcare Administration, Asia

University, Taichung, Taiwan
National Health Insurance Research Database, National Health Research Institutes, Taiwan on patients treated between 1999 and 2007. The study population comprised 1271,759 patients with T2DM without cirrhosis, 38,860 patients with cirrhosis without T2DM, 11,487 patients with T2DM and cirrhosis, and 579,173 patients without T2DM and cirrhosis (non-cirrhotic control group).

Results: The average incidence of dialysis in patients with T2DM and liver cirrhosis (2.466\%) was 10.6-, 2.3-, and 102.7-fold higher than that in patients with T2DM without cirrhosis $(0.232 \%)$, with cirrhosis without T2DM (1.071\%), and patients without both T2DM and cirrhosis (0.024\%), respectively. Adjusted odds ratio (OR) for dialysis risk was 3.19 in patients with T2DM and cirrhosis, 2.16 in patients with T2DM without cirrhosis, and 1.98 in patients with cirrhosis without T2DM, compared to that in patients

P.-T. Kung

Department of Medical Research, China Medical University Hospital-China Medical University, Taichung, Taiwan

W. H.-H. Sheu

Department of Medicine, School of Medicine, National Yang-Ming University, Taipei, Taiwan

W. H.-H. Sheu

Institute of Medical Technology, College of Life Science, National Chung-Hsing University,

Taichung, Taiwan

W. H.-H. Sheu

School of Medicine, National Defense Medical

Center, Taipei, Taiwan 
without T2DM and cirrhosis. Male sex (adjusted hazard ratio [HR] 1.15), age (45-49 vs. 20-34 years [reference]; adjusted HR 1.34), lowincome households (adjusted HR 1.46), cirrhosis (adjusted HR 3.42), and diabetic complications severity index (adjusted HR 1.71) were predictors of dialysis in T2DM patients. In addition, those with T2DM participating in the pay-for-performance (P4P) program had a significantly lower relative risk for requiring dialysis (HR 0.64).

Conclusion: Liver cirrhosis is an independent risk factor for dialysis in patients with T2DM. Participating in the P4P program for diabetes care may reduce the risk of requiring dialysis in patients with T2DM.

Keywords: Dialysis; Liver cirrhosis; Type 2 diabetes

\section{Key Summary Points}

Why carry out the study?

The incidence and prevalence of end-stage renal disease requiring dialysis in Taiwanese patients with diabetes have been reported to be the highest in the world.

Renal function impairment is one of the complications of liver cirrhosis.

We investigated the hypothesis that cirrhosis is a risk factor for dialysis in patients with type 2 diabetes mellitus (T2DM).

\section{What was learned from the study?}

The results revealed that cirrhosis significantly increased the risk of requiring dialysis in T2DM patients in Taiwan.

In addition to traditional chronic complications of diabetes, it is necessary to screen for liver disease more aggressively to avoid disease progression and the subsequent increased risk of renal failure requiring long-term dialysis.

Participating in the pay-for-performance program for diabetes care may reduce the risk of requiring dialysis in patients with T2DM.

\section{DIGITAL FEATURES}

This article is published with digital features to facilitate understanding of the article. To view digital features for this article go to https://doi. org/10.6084/m9.figshare.12866273.

\section{INTRODUCTION}

The prevalence of diabetes worldwide among adults has doubled since 1980, with type 2 diabetes mellitus (T2DM) being the most common form of diabetes [1-3]. In Taiwan, the prevalence is $11.8 \%$ among individuals aged $>$ 18 years (adults; males 13.1\%, females 10.5\%) [4]. The incidence and prevalence of end stage renal disease (ESRD) requiring persistent dialysis in Taiwanese patients with diabetes has been reported to be among the highest in the world [2]. Diabetic patients with the added complication of nephropathy have higher mortality rates than those without nephropathy $[5,6]$. Renal failure is one of the most common causes of death among diabetic patients, while poor glycemic control is associated with a shortened lifespan among diabetic patients, especially those with diabetic nephropathy [7].

Renal function impairment is one of the complications of liver cirrhosis. Both liver and kidney disease are associated with an increased risk of mortality in patients with diabetes $[8,9]$. Many studies have confirmed the relationships between chronic liver disease, liver cirrhosis, and glucose abnormalities [10, 11]. The liver plays a key role in the pathophysiology of diabetes. A large proportion of patients with cirrhosis have insulin resistance, and diabetes is common in patients with liver disease [12-14]. Diabetes, chronic liver disease and cirrhosis, and kidney disease ranked fifth, tenth, and ninth, respectively, among the ten leading causes of death in Taiwan in 2017; therefore, we cannot ignore the effect of these three interrelated diseases on the health of Taiwanese people [15].

The pathogenesis and prevention of T2DMrelated nephropathy and the mechanisms of reciprocal causation between chronic liver disease and diabetes have been studied. However, 
few studies have investigated the incidence of ESRD and persistent dialysis in patients with diabetes, with and without cirrhosis. Therefore, the aim of our study was to investigate the impact of liver cirrhosis on the incidence of dialysis among patients with T2DM. We hypothesized that cirrhosis is a risk factor for dialysis in patients with T2DM.

\section{METHODS}

\section{Data Source}

This study was based on data collected by the National Health Insurance Research Database (NHIRD), which falls within the framework of the National Health Insurance (NHI) program of the National Health Insurance Administration, Ministry of Health and Welfare and managed by the National Health Research Institutes under license; consequently, data cannot be made freely available. Requests for access to data in the NHIRD should be made to the National Health Research Institutes, Taiwan. Over 99\% of Taiwanese citizens have been enrolled in the NHI program since 1995.

The database used in this study consisted of two data subsets from the NHIRD. One was the longitudinal cohort of a specific patient database that contained the original claim data of Taiwanese patients with diabetes mellitus or cirrhosis or those who were receiving dialysis treatment. The second was the longitudinal health insurance database (LHID) of one million people randomly selected from the NHI registry for beneficiaries in Taiwan. Both data subsets comprised data on demographic characteristics and detailed clinical records, including age, sex, premium-based salary, outpatient visits, diagnostic codes, and admission records. All personal identification data were encrypted and anonymous. This study was approved by the research ethics committee of China Medical University and Hospital (IRB Number: CMUH103-REC-003).

\section{Study Subjects}

The study included adults aged $>20$ years who were divided into four groups: Group 1, patients with T2DM without cirrhosis; Group 2, patients with cirrhosis without T2DM; Group 3, patients with T2DM and cirrhosis; and Group 4, patients without T2DM and cirrhosis (non-cirrhotic control group). Group 4 was the reference group, and patients included in this group were recruited from the LHID, while patients in the three other groups were enrolled from a particular patient database. In order to reduce the study bias and focus on the effects of cirrhosis on dialysis among patients with T2DM, Group 3 only comprised patients who had developed cirrhosis after being diagnosed with T2DM.

Patients with T2DM were defined as those who had been hospitalized once or had made more than three outpatient visits within a 365-day period and who had been diagnosed with diabetes based on the following International Classification of Diseases, Ninth Revision (ICD-9) diagnosis codes: 250, 250.00, and 250.02. Patients with type 1 T2DM (T1DM) (ICD-9 codes: 250.01, 250.03), gestational diabetes mellitus (ICD-9 codes: 648.0, 648.8), neonatal diabetes mellitus (ICD-9 code: 775.1), and abnormal glucose tolerance test results (ICD-9 code: 790.2) were excluded. Patients on dialysis were identified as those who had a chronic kidney disease diagnosed with ICD-9 code 585 and had received continuous dialysis treatment for $>3$ months from 1998 to 2007 (including hemodialysis, peritoneal dialysis, and alternating hemodialysis or peritoneal dialysis). The date of diagnosis was defined as the date of the first dialysis. Patients who needed dialysis for acute renal failure were excluded. Patients with cirrhosis were those defined as being hospitalized once or who had more than three outpatient visits within a 365-day period and who had been diagnosed with cirrhosis according to ICD-9 diagnosis codes 571.2, 571.5, and 571.6. We excluded patients with a cirrhosis diagnosis or on dialysis prior to the study to reduce bias. Figure 1 shows the flowchart of patient selection for inclusion. 


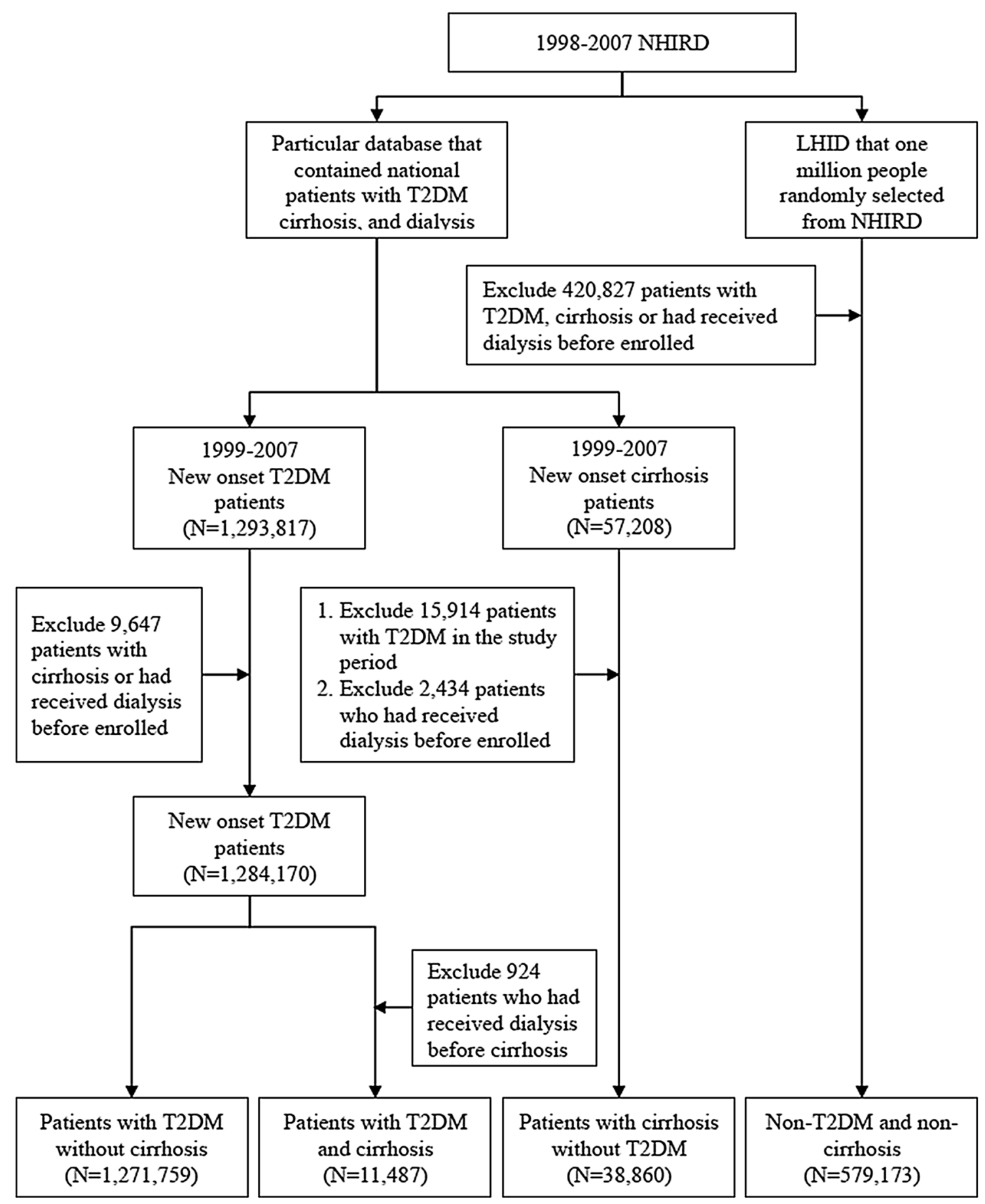

Fig. 1 Flowchart of the study subject selection process. NHIRD National Health Insurance Research Database, LHID Longitudinal Health Insurance Database, T2DM type 2 diabetes mellitus 


\section{Study Design}

This study was conducted using 1998-2007 claim data in the NHIRD and enrolled patients as study subjects from 1999 to 2007 to ensure the exclusion condition. The exclusion period for each participant was the year before enrollment in the study. For example, when patients were enrolled in 1999, the exclusion period was 1998. As a first step, we evaluated the different risks and related factors for dialysis between the four groups. Second, we evaluated the impact of cirrhosis on those on dialysis with T2DM. Each patient with T2DM was followed up until the date of incident dialysis or the end of 2007, whichever occurred first. Patients with ESRD requiring persistent dialysis were defined as those who continuously received hemodialysis and/or peritoneal dialysis for $>3$ months. The date of diagnosis was defined as the first date of receiving dialysis.

The control variables were sex, age, premium-based salary, low-income household, urbanization of residence area, Charlson Comorbidity Index (CCI), and Diabetic Complications Severity Index (DCSI). Premiumbased salaries of those included in the study were divided into eight groups according to the payroll bracket table of the National Health Insurance Administration, Taiwan. Low-income household status was derived from the registry of beneficiaries files from the study database, which means that the income of these patients was less than twofold the poverty threshold [16]. Insured dependents refers to those who have no income from salaries and wages and are financially dependent on family members. For example, an unemployed spouse or children are the health-insured dependents in a family.

Residential locations were determined according to seven levels of urbanization (a: highly urbanized township; b: moderately urbanized township; c: developing township; d: general township; e: aging township; f: agricultural township; g: rural area) and, based on urbanization characteristics, re-classified into four levels, with level 1 including a; level 2 including $\mathrm{b}+\mathrm{c}$; level 3 including $\mathrm{d}$; and level 4 including $\mathrm{e}+\mathrm{f}+\mathrm{g}$ [17]. The CCI was designed to be used with medical records for research relying on ICD-9-Clinical Modification (ICD-9$\mathrm{CM}$ ) diagnosis and procedure codes. This index is widely used in studies of disease outcome and resource use employing administrative databases. Further developed by Deyo et al., the CCI includes comorbidities such as myocardial infarct, congestive heart failure, peripheral vascular disease, cerebrovascular disease, dementia, chronic pulmonary disease, connective tissue disease, rheumatologic disease, ulcer disease, mild liver disease, hemiplegia, renal disease, tumor, malignancy, etc. The severity of comorbidities is scored as $\leq 1,2,3, \geq 4$, in which a higher score represents increased disease severity [18]. Complications of diabetes were classified and scored based on the DCSI published by Young et al., which includes retinopathy, nephropathy, neuropathy, cerebrovascular, cardiovascular and peripheral vascular disease, and metabolic complications identified by ICD9CM, from which, we excluded nephropathy because it was the endpoint of our study [19]. In addition to "with or without complications," the DCSI was calculated according to the severity score of complications $(0,1$ or 2 points). If a patient had such complications, the patient scored 1 more point in DCSI; if the complications were severe, the score was 2 points. The DCSI is a 13-point scale; however, nephropathy (score 2 points) was excluded in the present study. Therefore, the highest achievable score was 11 . Taiwan pay-for-performance (P4P) is designed as an integrated team-care program with team members, including doctors, nurses, dietitians, and other paramedical workers. The program offers financial rewards to doctors, partly based on the outcome of the patients. Diabetic patients who join the program need to undergo physical examinations, biochemical tests, and health education sessions regularly. In addition to the regular follow up by a medical team, P4P programs offer self-management education and support and are aimed to avoid subsequent complications of diabetes. 


\section{Statistical Analysis}

The software used in the present study was SAS version 9.1 (SAS Institute, Cary, NC, USA). Frequency distribution and percentages were used to present descriptive statistics, while means, standard deviation, frequencies, and percentage distribution were used to describe overall characteristics. A Chi-square test was used to analyze the relationship between each variable and dialysis. Logistic regression was used to analyze the correlation of different groups and incident dialysis in order to calculate the odds ratios (OR), after controlling for sex, age, premiumbased salary, low-income household, urbanization, and CCI. Cox proportional hazard models were used to investigate risks for requiring dialysis in patients with T2DM after controlling for sex, age, premium-based salary, low-income household, urbanization, and the DCSI. Statistical significance was defined as $p<0.05$.

\section{RESULTS}

\section{The Study Group Comprising Patients with T2DM and Cirrhosis had the Highest Proportion of Patients Undergoing Dialysis}

The characteristics of each study group are presented in Table 1. After exclusion, the total number of patients with T2DM without cirrhosis included in the study was 1271,759 , of whom 17,331 required dialysis therapy. Among patients with cirrhosis without T2DM, 37,662 did not undergo dialysis before the end of the observation period; however, 1198 patients received dialysis therapy. Among patients with T2DM and cirrhosis, dialysis was not needed by 10,708 patients; however, 779 patients received dialysis therapy. A total of 579,173 patients were included in the group without T2DM or cirrhosis, of whom 577,151 patients did not undergo dialysis, and 2022 patients required dialysis therapy before the end of the observation period.

\section{Trends Among Patients Requiring Long- Term Dialysis}

The trends in patients who required long-term dialysis among the four study groups in Taiwan from 1999 to 2007 are shown in Fig. 2. The highest incidence of patients who required dialysis occurred among those with T2DM and liver cirrhosis (average incidence 2.466\%) and the lowest incidence occurred among patients without T2DM or cirrhosis (average incidence $0.024 \%$ ). The average incidence of patients who required dialysis in the cirrhosis without T2DM and T2DM without cirrhosis groups were 1.071 and $0.232 \%$, respectively. This trend in the incidence of patients who required dialysis in the four groups remained unchanged during the 10-year observation period. Also, this trend in the incidence of patients requiring dialysis remained unchanged continuously during the observation period (Fig. 2; Electronic Supplementary Material Table S1).

\section{Risk Factors for Dialysis Requirement}

Table 2 shows the results of the logistic regression on the risks for requiring dialysis in each group. Multivariate logistic regression analysis was used to adjust for sex, age, premium-based salary, low-income households, urbanization, and the CCI. Compared with patients without T2DM or cirrhosis, for patients with T2DM and cirrhosis the unadjusted OR was 20.77 (95\% confidence interval [CI] 19.08-22.60, $p<0.001$ ) and the adjusted OR was 3.19 (95\% CI $2.92-3.48, p<0.001)$; in patients with T2DM without cirrhosis the unadjusted OR was 3.94 (95\% CI 3.77-4.13, $p<0.001)$ and the adjusted OR was 2.16 (95\% CI 2.05-2.27, $p<0.001)$; and in patients with cirrhosis without T2DM, the unadjusted OR was 9.08 (95\% CI 8.45-9.76, $p<0.001)$ and the adjusted OR was $1.98(95 \%$ CI 1.84-2.14, $p<0.001)$. The comparison of unadjusted OR to adjusted OR revealed that before adjustment, the study groups in descending order of incidence of patients requiring dialysis were T2DM with cirrhosis, cirrhosis without T2DM, and T2DM without cirrhosis. However, after adjustment, the 
Table 1 Characteristics of study groups

\begin{tabular}{|c|c|c|c|c|c|c|c|c|}
\hline \multirow[t]{2}{*}{ Variables $^{\mathrm{a}}$} & \multicolumn{2}{|c|}{$\begin{array}{l}\text { T2DM and } \\
\text { cirrhosis }\end{array}$} & \multicolumn{2}{|c|}{$\begin{array}{l}\text { T2DM without } \\
\text { cirrhosis }\end{array}$} & \multicolumn{2}{|c|}{$\begin{array}{l}\text { Cirrhosis without } \\
\text { T2DM }\end{array}$} & \multicolumn{2}{|c|}{$\begin{array}{l}\text { No T2DM and no } \\
\text { cirrhosis }\end{array}$} \\
\hline & $\bar{N}$ & $\%$ & $N$ & $\%$ & $N$ & $\%$ & $n$ & $\%$ \\
\hline Total & 11,487 & 100.00 & $1,271,759$ & 100.00 & 38,860 & 100.00 & 579,173 & 100.00 \\
\hline \multicolumn{9}{|l|}{ Dialysis } \\
\hline Yes & 779 & 6.78 & 17,331 & 1.36 & 1198 & 3.08 & 2022 & 0.35 \\
\hline No & 10,708 & 93.22 & $1,254,428$ & 98.64 & 37,662 & 96.92 & 577,151 & 99.65 \\
\hline \multicolumn{9}{|l|}{ Sex } \\
\hline Female & 3422 & 29.79 & 599,420 & 47.13 & 9760 & 25.12 & 283,925 & 49.02 \\
\hline Male & 8065 & 70.21 & 672,339 & 52.87 & 29,100 & 74.88 & 295,248 & 50.98 \\
\hline Age (years) ${ }^{b}$ & \multicolumn{2}{|c|}{$65.18 \pm 11.71$} & \multicolumn{2}{|c|}{$62.72 \pm 13.88$} & \multicolumn{2}{|c|}{$62.53 \pm 13.66$} & \multicolumn{2}{|c|}{$49.58 \pm 15.29$} \\
\hline $20-34$ & 48 & 0.42 & 28,823 & 2.27 & 908 & 2.34 & 100,020 & 17.27 \\
\hline $35-39$ & 153 & 1.33 & 35,502 & 2.79 & 1360 & 3.50 & 84,277 & 14.55 \\
\hline $40-44$ & 333 & 2.90 & 65,916 & 5.18 & 2337 & 6.01 & 81,683 & 14.10 \\
\hline $45-49$ & 721 & 6.28 & 107,593 & 8.46 & 3323 & 8.55 & 76,077 & 13.14 \\
\hline $50-54$ & 1165 & 10.14 & 151,257 & 11.89 & 3910 & 10.06 & 63,456 & 10.96 \\
\hline $55-59$ & 1430 & 12.45 & 177,438 & 13.95 & 4284 & 11.02 & 47,341 & 8.17 \\
\hline $60-64$ & 1552 & 13.51 & 140,995 & 11.09 & 4475 & 11.52 & 27,357 & 4.72 \\
\hline $65-69$ & 1860 & 16.19 & 149,541 & 11.76 & 5219 & 13.43 & 26,061 & 4.50 \\
\hline$\geq 70$ & 4225 & 36.78 & 414,693 & 32.61 & 13,044 & 33.57 & 72,885 & 12.58 \\
\hline Missing data & & & 1 & & & & 16 & \\
\hline \multicolumn{9}{|c|}{ Premium-based salary (NT\$) } \\
\hline Insured dependent & 3512 & 30.60 & 378,344 & 29.79 & 10,396 & 26.77 & 99,815 & 17.23 \\
\hline$\leq 17,280$ & 2441 & 21.27 & 263,778 & 20.77 & 8619 & 22.19 & 141,358 & 24.41 \\
\hline $17,281-22,800$ & 4219 & 36.76 & 382,448 & 30.12 & 14,308 & 36.84 & 158,442 & 27.36 \\
\hline $22,801-36,300$ & 685 & 5.97 & 121,768 & 9.59 & 2672 & 6.88 & 78,258 & 13.51 \\
\hline $36,301-45,800$ & 388 & 3.38 & 62,863 & 4.95 & 1557 & 4.01 & 43,944 & 7.59 \\
\hline $45,801-57,800$ & 73 & 0.64 & 19,218 & 1.51 & 555 & 1.43 & 24,120 & 4.16 \\
\hline $57,801-72,800$ & 104 & 0.91 & 22,042 & 1.74 & 452 & 1.16 & 18,517 & 3.20 \\
\hline$>72,800$ & 56 & 0.49 & 19,400 & 1.53 & 278 & 0.72 & 14,718 & 2.54 \\
\hline Missing data & 9 & & 1898 & & 23 & & 1 & \\
\hline \multicolumn{9}{|c|}{ Low-income household } \\
\hline Yes & 217 & 1.89 & 18,573 & 1.46 & 756 & 1.95 & 4599 & 0.79 \\
\hline No & 11,270 & 98.11 & $1,253,186$ & 98.54 & 38,104 & 98.05 & 574,574 & 99.21 \\
\hline
\end{tabular}


Table 1 continued

\begin{tabular}{|c|c|c|c|c|c|c|c|c|}
\hline \multirow[t]{2}{*}{ Variables $^{a}$} & \multicolumn{2}{|c|}{$\begin{array}{l}\text { T2DM and } \\
\text { cirrhosis }\end{array}$} & \multicolumn{2}{|c|}{$\begin{array}{l}\text { T2DM without } \\
\text { cirrhosis }\end{array}$} & \multicolumn{2}{|c|}{$\begin{array}{l}\text { Cirrhosis without } \\
\text { T2DM }\end{array}$} & \multicolumn{2}{|c|}{$\begin{array}{l}\text { No T2DM and no } \\
\text { cirrhosis }\end{array}$} \\
\hline & $N$ & $\%$ & $N$ & $\%$ & $N$ & $\%$ & $n$ & $\%$ \\
\hline \multicolumn{9}{|l|}{ Urbanization } \\
\hline 1 & 2580 & 22.57 & 348,758 & 27.50 & 8954 & 23.17 & 180,368 & 31.16 \\
\hline 2 & 4922 & 43.06 & 574,096 & 45.26 & 17,000 & 43.98 & 272,422 & 47.06 \\
\hline 3 & 2028 & 17.74 & 193,297 & 15.24 & 6790 & 17.57 & 75,576 & 13.06 \\
\hline 4 & 1901 & 16.63 & 152,226 & 12.00 & 5908 & 15.29 & 50,505 & 8.72 \\
\hline Missing data & 56 & & 3382 & & 208 & & 302 & \\
\hline \multicolumn{9}{|l|}{ CCI } \\
\hline$\leq 1$ & 744 & 6.48 & 780,538 & 61.37 & 3949 & 10.16 & 478,570 & 82.63 \\
\hline 2 & 1373 & 11.95 & 184,657 & 14.52 & 13,198 & 33.96 & 56,179 & 9.70 \\
\hline 3 & 2492 & 21.69 & 182,424 & 14.34 & 8796 & 22.64 & 23,963 & 4.14 \\
\hline$\geq 4$ & 6878 & 59.88 & 124,140 & 9.76 & 12,917 & 33.24 & 20,461 & 3.53 \\
\hline
\end{tabular}

CCI Charlson Comorbidity Index, NT\$ New Taiwan Dollar, T2DM type 2 diabetes mellitus

a See section Study Design for detailed description of the variables

b Mean \pm standard deviation $(\mathrm{SD})$

adjusted OR in patients with cirrhosis without T2DM was lower than that in patients with T2DM without cirrhosis. The adjusted OR of dialysis in patients with low-income households was 1.37 (95\% CI 1.25-1.51, $p<0.001$ ) compared to patients with non-low-income households. The results of the analysis of premium-based salary showed that insured dependents and those who had lower premium-based salaries had higher adjusted ORs for dialysis. In contrast, those with higher premium-based salaries had lower adjusted ORs for dialysis $(p<0.05)$. The adjusted ORs of dialysis was significantly higher in high-level urbanization areas compared with low-level ones $(p<0.05)$.

\section{Relative Risks and Related Factors for Dialysis}

Table 3 shows the adjusted hazard ratio (HR) and 95\% CI for dialysis in patients with T2DM. In accordance with the analytical methods used for diabetic complications, Model I and Model II were used to present the results. In Model I, the categorical variables were types of diabetic complications, while in Model II, diabetic complications were continuous variables calculated using the DCSI.

Model I Diabetic complications were classified by types in Model I. Adjusted HR in male patients was 1.16 (95\% CI 1.13-1.20); further, patients who were 45-49 years old had the highest adjusted HR (1.33, 95\% CI 1.15-1.53) compared with patients who were $20-34$ years old. When a premium-based salary of $\leq 17,280$ was used as the reference group, insured dependents had the highest adjusted HR. Lowincome households were found to a risk factor for dialysis (adjusted HR 1.44, 95\% CI 1.30-1.59). The relative risks for requiring dialysis in the population living in cities with higher levels of urbanization were $>1$ $(p<0.05)$. With regards to diabetes complications, compared with patients without complications, the adjusted HR for dialysis in descending order were as follows: peripheral vascular disease $(3.54,95 \%$ CI 3.39-3.69), 


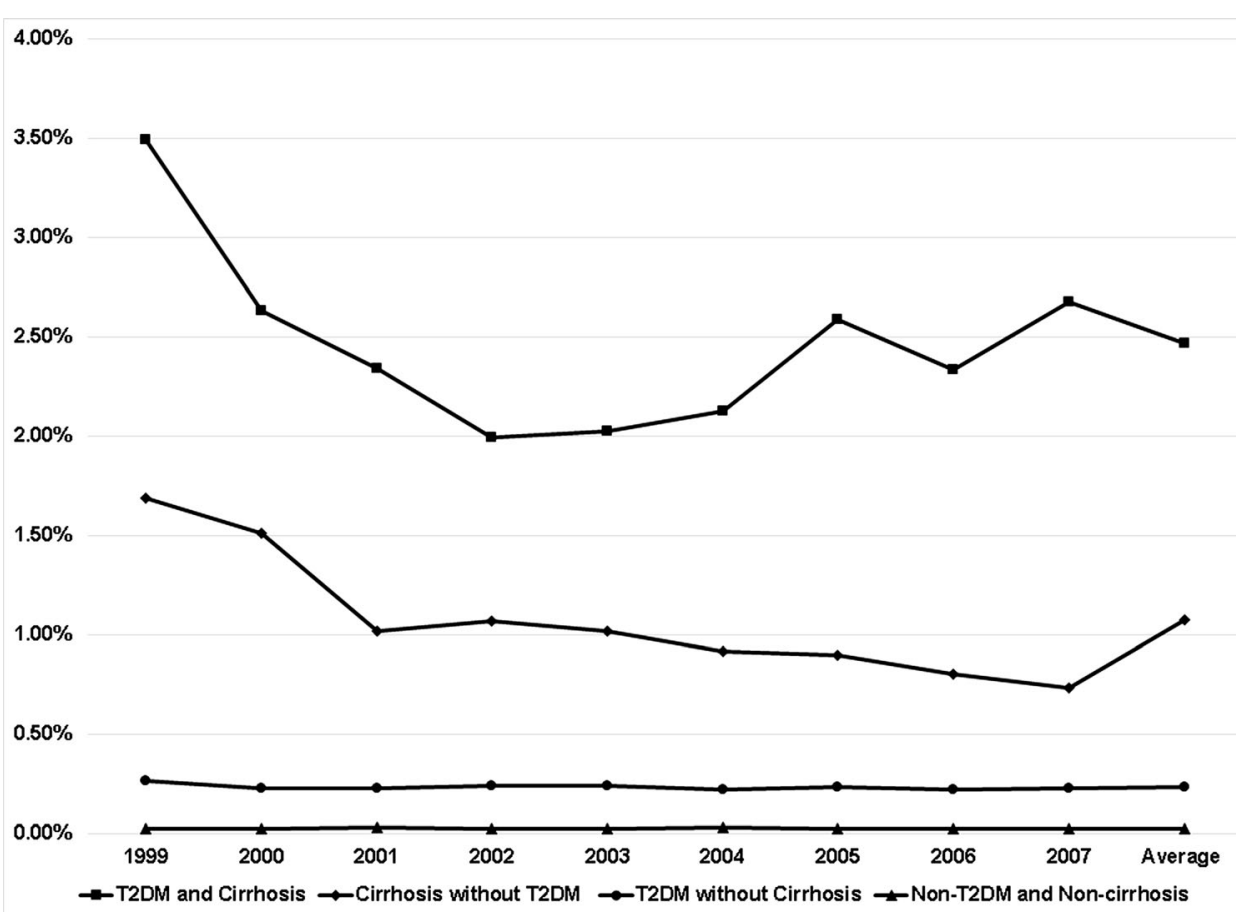

Fig. 2 Trend in the incidence of continuous dialysis among the four study groups from 1999 to 2007 T2DM type 2 diabetes mellitus

metabolic complications (3.06, 95\% CI $2.75-3.42)$, cerebrovascular disease $(2.06,95 \%$ CI 1.94-2.18), cardiovascular disease $(2.04,95 \%$ CI 1.93-2.17), and neuropathy $(1.71,95 \%$ CI 1.63-1.79). Regarding the role of cirrhosis in the need for long-term dialysis in patients with T2DM, when using non-cirrhosis patients as a reference group, the adjusted HR of dialysis in patients with cirrhosis was $3.30 \quad(95 \%$ CI 3.07-3.55), ranking second to that in patients with diabetic peripheral vascular disease (adjusted HR 3.54). When using patients not participating in the $\mathrm{P} 4 \mathrm{P}$ program for diabetes care as a reference group, those with T2DM participating in the P4P program had a lower relative risk for requiring dialysis (HR 0.64, 95\% CI 0.61-0.67). The adjusted HR of diabetic retinopathy was 0.54 (95\% CI 0.48-0.60).

Model II In Model II, T2DM complications were calculated according to the DCSI. The findings of sex, age, and socioeconomic conditions were consistent with Model I. With respect to severity of diabetic complications, when using patients without complications as a reference group, the adjusted HR increased by 1.71-fold (95\% CI 1.68-1.75) for each additional level of DCSI severity. Regarding the role of cirrhosis in determining requirements for longterm dialysis in patients with T2DM, when using patients without liver cirrhosis as a reference group, the adjusted HR in patients with cirrhosis was 3.42 (95\% CI 3.18-3.68). The adjusted HR in diabetic complication scores was 1.71. When using patients not participating in the $\mathrm{P} 4 \mathrm{P}$ program for diabetes care as a reference group, the relative risk for requiring dialysis in T2DM patients participating in the $\mathrm{P} 4 \mathrm{P}$ program fell to 0.63 (95\% CI 0.60-0.65).

\section{DISCUSSION}

\section{Trends in the Incidence of Dialysis Among Patients with Diabetes and/or Cirrhosis}

The results of the present study indicate that the incidence of patients who progressed to ESRD and required persistent dialysis was significantly 
Table 2 Relative risks and related factors for dialysis using logistic regression analysis

\begin{tabular}{|c|c|c|c|c|c|c|}
\hline \multirow[t]{2}{*}{ Variables } & \multicolumn{3}{|c|}{ Unadjusted } & \multicolumn{3}{|c|}{ Adjusted } \\
\hline & $\overline{\text { OR }}$ & $95 \% \mathrm{CI}$ & $p$ value & $\overline{\mathrm{OR}}$ & $95 \% \mathrm{CI}$ & $p$ value \\
\hline \multicolumn{7}{|l|}{ Groups } \\
\hline T2DM and cirrhosis & 20.77 & $19.08-22.6$ & $<0.001$ & 3.19 & $2.92-3.48$ & $<0.001$ \\
\hline T2DM without cirrhosis & 3.94 & $3.77-4.13$ & $<0.001$ & 2.16 & $2.05-2.27$ & $<0.001$ \\
\hline Cirrhosis without T2DM & 9.08 & $8.45-9.76$ & $<0.001$ & 1.98 & $1.84-2.14$ & $<0.001$ \\
\hline \multicolumn{7}{|c|}{ Non-T2DM and non-cirrhotic (reference) } \\
\hline \multicolumn{7}{|l|}{ Sex } \\
\hline \multicolumn{7}{|l|}{ Female (reference) } \\
\hline Male & 1.11 & $1.08-1.14$ & $<0.001$ & 1.01 & $0.98-1.03$ & 0.746 \\
\hline \multicolumn{7}{|l|}{ Age (years) } \\
\hline \multicolumn{7}{|l|}{ 20-34 (reference) } \\
\hline $35-39$ & 1.38 & $1.22-1.57$ & $<0.001$ & 0.98 & $0.86-1.11$ & 0.709 \\
\hline $40-44$ & 2.35 & $2.10-2.62$ & $<0.001$ & 1.16 & $1.04-1.30$ & 0.011 \\
\hline $45-49$ & 3.24 & $2.92-3.59$ & $<0.001$ & 1.21 & $1.09-1.35$ & $<0.001$ \\
\hline $50-54$ & 3.83 & $3.46-4.24$ & $<0.001$ & 1.16 & $1.05-1.29$ & 0.005 \\
\hline $55-59$ & 3.58 & $3.23-3.96$ & $<0.001$ & 0.89 & $0.80-0.99$ & 0.028 \\
\hline $60-64$ & 4.55 & $4.11-5.04$ & $<0.001$ & 0.89 & $0.80-0.99$ & 0.031 \\
\hline $65-69$ & 4.51 & $4.08-5.00$ & $<0.001$ & 0.74 & $0.67-0.83$ & $<0.001$ \\
\hline$\geq 70$ & 3.81 & $3.46-4.20$ & $<0.001$ & 0.57 & $0.51-0.63$ & $<0.001$ \\
\hline \multicolumn{7}{|l|}{ Premium-based salary (NT\$) } \\
\hline Insured dependent & 1.36 & $1.31-1.41$ & $<0.001$ & 1.23 & $1.18-1.28$ & $<0.001$ \\
\hline \multicolumn{7}{|l|}{$\leq 17,280 \mathrm{NT}$ (reference) } \\
\hline $17,281-22,800$ & 1.14 & $1.10-1,10$ & $<0.001$ & 1.12 & $1.07-1.17$ & $<0.001$ \\
\hline $22,801-36,300$ & 0.60 & $0.56-0.64$ & $<0.001$ & 0.66 & $0.61-0.70$ & $<0.001$ \\
\hline $36,301-45,800$ & 0.53 & $0.48-0.57$ & $<0.001$ & 0.60 & $0.55-0.65$ & $<0.001$ \\
\hline $45,801-57,800$ & 0.32 & $0.28-0.38$ & $<0.001$ & 0.49 & $0.41-0.57$ & $<0.001$ \\
\hline $57,801-72,800$ & 0.40 & $0.35-0.46$ & $<0.001$ & 0.51 & $0.44-0.60$ & $<0.001$ \\
\hline$>72,800$ & 0.26 & $0.22-0.32$ & $<0.001$ & 0.36 & $0.29-0.44$ & $<0.001$ \\
\hline
\end{tabular}

Low-income household

No (reference)

\begin{tabular}{lllllll} 
Yes & 1.98 & $1.82-2.17$ & $<0.001$ & 1.37 & $1.25-1.51$ & $<0.001$ \\
\hline
\end{tabular}


Table 2 continued

\begin{tabular}{|c|c|c|c|c|c|c|}
\hline \multirow[t]{2}{*}{ Variables } & \multicolumn{3}{|c|}{ Unadjusted } & \multicolumn{3}{|c|}{ Adjusted } \\
\hline & $\overline{\mathrm{OR}}$ & $95 \% \mathrm{CI}$ & $p$ value & $\overline{\mathrm{OR}}$ & $95 \% \mathrm{CI}$ & $p$ value \\
\hline \multicolumn{7}{|c|}{ Urbanizations } \\
\hline 1 & 0.88 & $0.84-0.93$ & $<0.001$ & 1.17 & $1.11-1.23$ & $<0.001$ \\
\hline 2 & 0.96 & $0.92-1.01$ & 0.100 & 1.17 & $1.11-1.22$ & $<0.001$ \\
\hline 3 & 0.98 & $0.93-1.03$ & 0.354 & 1.05 & $0.99-1.10$ & 0.097 \\
\hline \multicolumn{7}{|c|}{4 (reference) } \\
\hline \multicolumn{7}{|l|}{ CCI } \\
\hline \multicolumn{7}{|c|}{$\leq 1$ (reference) } \\
\hline 2 & 5.98 & $5.69-6.28$ & $<0.001$ & 5.68 & $5.40-5.97$ & $<0.001$ \\
\hline 3 & 9.38 & $8.95-9.83$ & $<0.001$ & 8.25 & $7.86-8.65$ & $<0.001$ \\
\hline$\geq 4$ & 28.98 & $27.80-30.20$ & $<0.001$ & 26.39 & $25.26-27.57$ & $<0.001$ \\
\hline
\end{tabular}

CI Confidence interval, $O R$ odds ratio

higher in those patients with T2DM and cirrhosis than in the other patient groups. This trend remained unchanged during the entire observation period. A previous study showed that approximately $26.2 \%$ of patients with T2DM in the USA had diabetic kidney disease, defined by albuminuria, reduced estimated glomerular filtration rate, or both [6]. In a study carried out in France, the incidence rate of dialysis was 18.4 per 1000 person-years in patients with T2DM [20]. Significant causal factors for the need for dialysis in Taiwan is the ageing population and a rapidly increasing rate of T2DM [2]. In 2010, diabetes accounted for 66 and $61 \%$ of patients with ESRD in Singapore and Malaysia, respectively. Korea, Hong Kong, Taiwan, and Japan reported rates of ESRD due to diabetic nephropathy of $>40 \%$ in 2012 [21]. According to US Renal Data System data, the proportion of ESRD patients with diabetes has stabilized and may even have declined in recent years [22]. However, the incidence of ESRD requiring dialysis among patients with T2DM and cirrhosis increased in the present study, while the incidence of dialysis in the other groups decreased during the observation period.

\section{Impact of Cirrhosis on the Incidence of Dialysis in T2DM}

Many studies have investigated the relationship between chronic liver disease, liver cirrhosis, and renal injury, and regardless of whether liver disease is infectious (especially hepatitis C) or non-infectious (such as non-alcoholic fatty liver disease), nephropathy is corroborated $[6,10,12]$. Acute kidney injury is known to occur in patients with advanced cirrhosis [23]. Although diabetic nephropathy complications are characterized by glomerular lesions and sclerosis [24], cirrhosis-related nephropathy is usually an acute renal injury with a major clinical presentation of renal tubular damage. Nephropathy in patients with cirrhosis and diabetes may progress to chronic kidney disease, acute kidney injury, even leading to mixed acute and chronic renal injuries that worsen and complicate the nephropathy. In the past, few studies have investigated the association with cirrhosis in patients with T2DM, and its impact on progression to ESRD requiring longterm dialysis therapy.

Between 1999 and 2007, the average incidence of dialysis among patients with T2DM 
Table 3 Relative risks and related factors for dialysis among patients with type 2 diabetes mellitus

\begin{tabular}{|c|c|c|c|c|c|c|}
\hline \multirow[t]{2}{*}{ Variables } & \multicolumn{3}{|l|}{ Model I } & \multicolumn{3}{|l|}{ Model II } \\
\hline & Adjusted HR & $95 \% \mathrm{CI}$ & $p$ value & Adjusted HR & $95 \% \mathrm{CI}$ & $p$ value \\
\hline \multicolumn{7}{|l|}{ Liver cirrhosis } \\
\hline \multicolumn{7}{|l|}{ No (reference) } \\
\hline Yes & 3.30 & $3.07-3.55$ & $<0.001$ & 3.42 & $3.18-3.68$ & $<0.001$ \\
\hline \multicolumn{7}{|l|}{ Sex } \\
\hline \multicolumn{7}{|l|}{ Female (reference) } \\
\hline Male & 1.16 & $1.13-1.2$ & $<0.001$ & 1.15 & $1.12-1.19$ & $<0.001$ \\
\hline \multicolumn{7}{|l|}{ Age (years) } \\
\hline \multicolumn{7}{|l|}{ 20-34 (reference) } \\
\hline $35-39$ & 1.12 & $0.94-1.32$ & 0.200 & 1.11 & $0.94-1.31$ & 0.234 \\
\hline $40-44$ & 1.29 & $1.12-1.5$ & 0.001 & 1.29 & $1.12-1.50$ & 0.001 \\
\hline $45-49$ & 1.33 & $1.15-1.53$ & $<0.001$ & 1.33 & $1.15-1.53$ & $<0.001$ \\
\hline $50-54$ & 1.30 & $1.13-1.49$ & $<0.001$ & 1.30 & $1.13-1.49$ & $<0.001$ \\
\hline $55-59$ & 0.94 & $0.82-1.08$ & 0.392 & 0.93 & $0.81-1.07$ & 0.335 \\
\hline $60-64$ & 0.95 & $0.82-1.09$ & 0.439 & 0.94 & $0.82-1.08$ & 0.402 \\
\hline $65-69$ & 0.81 & $0.71-0.94$ & 0.004 & 0.80 & $0.70-0.92$ & 0.002 \\
\hline$\geq 70$ & 0.63 & $0.55-0.72$ & $<0.001$ & 0.62 & $0.54-0.71$ & $<0.001$ \\
\hline \multicolumn{7}{|c|}{ Premium-based salary (NT\$) } \\
\hline Insured dependent & 1.22 & $1.17-1.27$ & $<0.001$ & 1.21 & $1.16-1.26$ & $<0.001$ \\
\hline \multicolumn{7}{|l|}{$\leq 17,280$ (reference) } \\
\hline $17,281-22,800$ & 1.11 & $1.06-1.16$ & $<0.001$ & 1.12 & $1.07-1.17$ & $<0.001$ \\
\hline $22,801-36,300$ & 0.59 & $0.55-0.64$ & $<0.001$ & 0.60 & $0.56-0.64$ & $<0.001$ \\
\hline $36,301-45,800$ & 0.55 & $0.5-0.61$ & $<0.001$ & 0.55 & $0.50-0.60$ & $<0.001$ \\
\hline $45,801-57,800$ & 0.41 & $0.34-0.49$ & $<0.001$ & 0.40 & $0.33-0.49$ & $<0.001$ \\
\hline $57,801-72,800$ & 0.45 & $0.38-0.53$ & $<0.001$ & 0.45 & $0.38-0.53$ & $<0.001$ \\
\hline$>72,800$ & 0.29 & $0.23-0.36$ & $<0.001$ & 0.28 & $0.22-0.35$ & $<0.001$ \\
\hline
\end{tabular}

Low-income household

No (reference)

Yes

1.44

$1.3-1.59$

$<0.001$

1.46

$1.33-1.62<0.001$ 
Table 3 continued

\begin{tabular}{|c|c|c|c|c|c|c|}
\hline \multirow[t]{2}{*}{ Variables } & \multicolumn{3}{|l|}{ Model I } & \multicolumn{3}{|l|}{ Model II } \\
\hline & Adjusted HR & $95 \% \mathrm{CI}$ & $p$ value & Adjusted HR & $95 \% \mathrm{CI}$ & $p$ value \\
\hline \multicolumn{7}{|l|}{ Urbanizations } \\
\hline 1 & 1.11 & $1.05-1.17$ & 0.001 & 1.09 & $1.03-1.15$ & 0.003 \\
\hline 2 & 1.15 & $1.1-1.21$ & $<0.001$ & 1.13 & $1.08-1.19$ & $<0.001$ \\
\hline 3 & 1.08 & $1.02-1.14$ & 0.009 & 1.07 & $1.01-1.13$ & 0.025 \\
\hline \multicolumn{7}{|l|}{4 (reference) } \\
\hline \multicolumn{7}{|l|}{ P4P } \\
\hline \multicolumn{7}{|l|}{ No (reference) } \\
\hline Yes & 0.64 & $0.61-0.67$ & $<0.001$ & 0.63 & $0.60-0.65$ & $<0.001$ \\
\hline \multicolumn{7}{|l|}{ Complications of diabetes } \\
\hline Retinopathy & 0.54 & $0.48-0.6$ & $<0.001$ & & & \\
\hline Neuropathy & 1.71 & $1.63-1.79$ & $<0.001$ & & & \\
\hline Cerebrovascular & 2.06 & $1.94-2.18$ & $<0.001$ & & & \\
\hline Cardiovascular & 2.04 & $1.93-2.17$ & $<0.001$ & & & \\
\hline Peripheral vascular disease & 3.54 & $3.39-3.69$ & $<0.001$ & & & \\
\hline Metabolic & 3.06 & $2.75-3.42$ & $<0.001$ & & & \\
\hline DCSI & & & & 1.71 & $1.68-1.75$ & $<0.001$ \\
\hline
\end{tabular}

DCSI Diabetes Complications Severity Index, $H R$ hazard ratio, $P 4 P$ pay-for-performance

and liver cirrhosis (2.466\%) was 10.6 times, 2.3 times, and 102.7 times higher than that among patients with T2DM without cirrhosis (0.232\%), cirrhosis without T2DM (1.071\%), and nonT2DM and non-cirrhosis (0.024\%), respectively. This trend in the incidence of patients who required dialysis remained unchanged during the ten-year observation period. The OR for requiring dialysis in patients with $\mathrm{T} 2 \mathrm{DM}$ and cirrhosis was the highest among the four groups in this study.

\section{Impact of Chronic Complications on the Incidence of Dialysis Among T2DM}

The logistic regression showed that patients in low-income households, insured dependents, and those with lower premium-based salary had a higher risk of requiring dialysis. According to a survey of living conditions among low-income households in 2008 from the Ministry of the Interior, Taiwan, $70.40 \%$ of heads of households did not complete junior high school education. The education level in low-income households may be lower than that in the general population, which provides evidence supporting the inferences stated above. The results of the Cox model indicate that male sex, low-income households, complications of diabetes, contained peripheral vascular disease, metabolic complications, cerebrovascular disease, cardiovascular disease and neuropathy, and cirrhosis were risk factors for dialysis in the patients with T2DM in our study. When complications of diabetes were analyzed according to the DCSI, their severity remained a risk factor for dialysis. An unexpected outcome of our 
analysis was that patients with diabetic retinopathy had a lower risk for requiring dialysis than patients without this microvascular complication. Possible reasons for this outcome are as follows. First, this study showed the diagnostic status of diseases, which differs from disease morbidity. The diagnosis of diabetic retinopathy is not as straightforward as the diagnosis of other diabetic complications. Diabetic retinopathy must be diagnosed via examination of the fundus, which is usually performed by ophthalmologists using an ophthalmoscope, while other complications can be diagnosed by internal or family medicine physicians. Furthermore, the early symptoms of diabetic retinopathy are not obvious, and its clinical presentations are not highly specific; thus, it can be easily overlooked unless the condition deteriorates acutely. In contrast, although neuropathy also occurs in the early stage of diabetes, its symptoms are often more obvious (e.g., peripheral numbness, tingling). Therefore, it is easier for physicians to diagnose diabetic neuropathy based on its symptomatic manifestations. As a result of these factors, screening rates for diabetic retinopathy may be low, resulting in an underestimation of diabetic retinopathy.

Previous studies have shown that diabetic nephropathy is associated with age, sex, and poor blood pressure and blood glucose control and that there is a relationship between blood glucose control and diabetic microvascular complications $[25,26]$. Diabetic nephropathy is closely associated with diabetic macrovascular complications as hyperglycemia is an important cerebrovascular risk factor that induces a variety of biochemical changes within endothelial cells in the cerebral vasculature [27]. T2DM is associated with at least a twofold increased risk for all diabetic macrovascular complications, coronary heart disease, stroke, and peripheral vascular disease [28]. Therefore, both complications and the severity of the disease are significantly associated with the need for dialysis in patients with T2DM. According to the results of the present study, in terms of the role of cirrhosis in the need for dialysis in patients with T2DM, the adjusted HR in patients with cirrhosis ranked second only to that in patients with diabetic peripheral vascular disease. In addition to traditional risk factors, cirrhosis is an important independent risk factor for dialysis in patients with T2DM.

\section{Impact of Joining the P4P Program for Diabetes Care on the Incidence of Dialysis in T2DM}

There was a $36 \%$ risk reduction in dialysis in T2DM patients who joined the P4P program for diabetes care. The P4P program for diabetes care was implemented in Taiwan in 2001. Studies have documented the effectiveness of the P4P program in improving essential clinical examinations and lowering the incidence of complications $[29,30]$. If problems related to screening and diagnosis rates are excluded, previous studies have also have demonstrated the inconsistency between diabetic retinopathy and nephropathy, which is consistent with the results of the present study. One previous study showed that diabetic retinopathy was not a predictive factor of nephropathy [31]. In contrast, diabetic retinopathy has been proposed as a predictor for diabetic nephropathy in a recent meta-analysis [32]. Whether genes and ethnicity play a role remains to be determined. Moreover, not all diabetic patients experience renal dysfunction due to diabetic nephropathy. The prevalence of non-diabetic renal disease (NDRD) in patients with diabetes is high, mainly presenting as nondiabetes-related glomerulonephritis or interstitial nephropathy [33-35].

The results of the present study may be explained by the low, even rare, prevalence of diabetic retinopathy in the patient population in this study and the short duration of diabetes. However, in order to deal with low screening and diagnosis rates for retinopathy, prospective clinical studies are required to investigate the association between diabetic retinopathy and nephropathy in T2DM in order to understand the underlying reasons and relevant mechanisms.

\section{Limitations}

There are a few limitations to this study that should be acknowledged in the present study. 
First, the NHIRD and the ICD-9 diagnosis codes were used to define relevant diseases. Thus, although no laboratory data were required for diagnosis, a relatively strict diagnostic definition was adopted. Second, patients who had been hospitalized once or had more than three outpatient visit in a 365-day period and who were diagnosed with diabetes or cirrhosis based on the ICD-9 diagnosis codes were included in this study. The definition of diabetes employed in previous studies in Taiwan was used [36]. A sample questionnaire survey asking patients about the status of their medication use and physicians' notifications were used as diagnostic criteria in a previous study and the consistency between ICD-9 codes and questionnaires verified the sensitivity $(96.9 \%)$ and positive predictive value $(93.9 \%)$ of this approach. Third, in the present study, the amount of insurance and urbanization levels of the region were used to estimate socioeconomic status and the urban-rural gap; however, the actual situation may be different. No record of patients' lifestyles, health behaviors, and clinical data was available in the NHIRD; thus, we were unable to draw relevant inferences based on disease mechanisms. Additionally, patients' medications were not analyzed in the present study.

\section{Strength of the Study}

The strength of the study is that it combined two large and representative nationwide databases in Taiwan to analyze the correlation between cirrhosis and dialysis among patients with T2DM, thereby avoiding bias from selection, non-response, or poor recall. Previous epidemiology studies have shown that the NHIRD has good levels of accuracy and completeness $[37,38]$. The association between the presence of cirrhosis and incident dialysis is present, even though only 10 years of data from the database were used; the study maintains the accuracy and representativeness of the database.

\section{CONCLUSIONS}

The results of the present study indicate that cirrhosis significantly increases the risk of the need for dialysis in T2DM patients. Between 1999 and 2007, the average incidence of dialysis was 10.2-fold greater in patients with diabetes and cirrhosis than in patients with T2DM without cirrhosis. After adjustment, the adjusted OR was significantly higher in patients with T2DM and cirrhosis than in patients with T2DM without cirrhosis. If T2DM patients have abnormal liver functions, it is necessary to prevent disease progression to cirrhosis, which leads to an increased risk of requiring dialysis. In addition to cirrhosis, risk factors for dialysis in T2DM patients also include male sex, older age, low-income households, and diabetic complications. The risk of requiring dialysis in T2DM patients with cirrhosis ranked second only to that in patients with diabetic peripheral vascular disease. Early and immediate prevention of renal disease requires intervention in populations at high risk of requiring dialysis to avoid progression to long-term dialysis treatment, which causes a significant burden on families and society and increases healthcare costs. Prospective clinical studies are required to further investigate the mechanisms and association between diabetic retinopathy and dialysis. According to the results of this study, participation in the P4P program for diabetes care may reduce the risk of requiring dialysis in patients with T2DM.

\section{ACKNOWLEDGEMENTS}

We appreciate the provision of research datasets from the Ministry of Health and Welfare. We are also grateful to Health Data Science Center, China Medical University Hospital for providing administrative, technical, and funding support.

Funding. The authors are grateful for financial support from the Asia University and China Medical University Hospital (Grant numbers: ASIA-108-CMUH-15). The Rapid Service Fee was funded by the authors.

Editorial Assistance. We would like to thank Nikita Pandey of Editage (https://www. 
editage.com) for English language editing. This assistance was funded by the authors.

Authorship. All named authors meet the International Committee of Medical Journal Editors (ICMJE) criteria for authorship for this article, take responsibility for the integrity of the work as a whole, and have given their approval for this version to be published.

Authorship Contributions. Y-JS made substantial contributions to the conception and design of the study, participated in drafting the article, and revised the article critically for important intellectual content. P-TK made substantial contributions to the interpretation and analysis of data. WH-HS participated in drafting the article and revised it critically for important intellectual content. W-YK and MHA made substantial contributions to the analysis and interpretation of data. W-CT made substantial contributions to the conception and design of the study, revised it critically for important intellectual content, and gave final approval of the version to be submitted and any revised version.

Disclosures. Yi-Jing Sheen, Pei-Tseng Kung, Wayne H.-H. Sheu, Wei-Yin Kuo, and WenChen Tsai have nothing to disclose.

Compliance with Ethics Guidelines. This study was approved by the research ethics committee of China Medical University and Hospital (IRB Number: CMUH103-REC-003).

Data Availability. The datasets generated during and/or analyzed during the current study are not publicly available for the following reasons. The NHIRD was provided by the National Health Insurance Administration, Ministry of Health and Welfare and managed by National Health Research Institutes under license and so cannot be made freely available. Requests for access to these data should be made to the National Health Research Institutes, Taiwan (https://www.nhird.nhri.org.tw/). We have added the description of the data in the Methods section.
Open Access. This article is licensed under a Creative Commons Attribution-NonCommercial 4.0 International License, which permits any non-commercial use, sharing, adaptation, distribution and reproduction in any medium or format, as long as you give appropriate credit to the original author(s) and the source, provide a link to the Creative Commons licence, and indicate if changes were made. The images or other third party material in this article are included in the article's Creative Commons licence, unless indicated otherwise in a credit line to the material. If material is not included in the article's Creative Commons licence and your intended use is not permitted by statutory regulation or exceeds the permitted use, you will need to obtain permission directly from the copyright holder. To view a copy of this licence, visit http://creativecommons.org/licenses/by$\mathrm{nc} / 4.0 /$.

\section{REFERENCES}

1. Roglic G. Global report on diabetes. Geneva: World Health Organization; 2016.

2. Wu MY, Wu MS. Taiwan renal care system: a learning health-care system. Nephrology. 2018;23: $112-5$.

3. Yuan H, Li X, Wan G, et al. Type 2 diabetes epidemic in East Asia: a 35-year systematic trend analysis. Oncotarget. 2018;9:6718-27.

4. Ministry of Health and Welfare. 2017 annual report: health promotion administration. Taiwan: Ying-Wei Wang; 2017. https://www.hpa.gov.tw/ File/Attach/8188/File_8122.pdf. Accessed 12 june 2020.

5. Lin YC, Hsu CY, Kao CC, et al. Incidence and prevalence of ESRD in Taiwan Renal Registry Data System (TWRDS). Acta Nephrol. 2014;28:65-8.

6. Afkarian M, Zelnick LR, Hall YN, et al. Clinical manifestations of kidney disease among US adults with diabetes, 1988-2014. JAMA. 2016;316:602-10.

7. Nakamura J, Kamiya H, Haneda M, et al. Causes of death in Japanese patients with diabetes based on the results of a survey of 45,708 cases during 2001-2010: report of the committee on causes of death in diabetes mellitus. J Diabetes Investig. 2017;8:397-410. 
8. Chen CW, Drechsler C, Suntharalingam P, Karumanchi SA, Wanner C, Berg AH. High glycated albumin and mortality in persons with diabetes mellitus on hemodialysis. Clin Chem. 2017;63: 477-85.

9. Shyu YC, Huang TS, Chien CH, Yeh CT, Lin CL, Chien RN. Diabetes poses a higher risk of hepatocellular carcinoma and mortality in patients with chronic hepatitis B: a population-based cohort study. J Viral Hepat. 2019;26:718-26.

10. Hazlehurst JM, Woods C, Marjot T, Cobbold JF, Tomlinson JW. Non-alcoholic fatty liver disease and diabetes. Metabolism. 2016;65:1096-108.

11. Jáquez Quintana JO, García-Compean D, González González JA, et al. The impact of diabetes mellitus in mortality of patients with compensated liver cirrhosis-a prospective study. Ann Hepatol. 2016;10:56-62.

12. Tilg H, Moschen AR, Roden M. NAFLD and diabetes mellitus. Nat Rev Gastroenterol Hepatol. 2017;14: 32.

13. Petrides AS, Stanley T, Matthews DE, Vogt C, Bush AJ, Lambeth H. Insulin resistance in cirrhosis: prolonged reduction of hyperinsulinemia normalizes insulin sensitivity. Hepatology. 1998;28:141-9.

14. Hsu CS, Kao JH. An update on non-alcoholic fatty liver disease and non-alcoholic steatohepatitis in Asia. Expert Rev Gastroenterol Hepatol. 2017;11: 759-72.

15. Ministry of Health and Welfare, Taiwan. Health and welfare report 2017. Taipei City: Ministry of Health and Welfare; 2018.

16. National Health Insurance Administration, Taiwan. Payroll bracket table 1999 2007. https://www.nhi. gov.tw/Content_List.aspx?n=1269AC29FCEF36A3 \&topn=3185A4DF68749BA9. Accessed 12 Feb 2020.

17. Liu CY, Hung YT, Chuang YL, Chen YJ, Weng WS, Liu JS. Incorporating development stratification of Taiwan townships into sampling design of large scale health interview survey. J Health Manag. 2006;4:1-22. (in Chinese). https://doi.org/10. 29805/JHM.200606.0001.

18. Deyo RA, Cherkin DC, Ciol MA. Adapting a clinical comorbidity index for use with ICD-9-CM administrative databases. J Clin Epidemiol. 1992;45: 613-9.

19. Young BA, Lin E, Von Korff M, et al. Diabetes complications severity index and risk of mortality, hospitalization, and healthcare utilization. Am J Manag Care. 2008;14:15-23.
20. Hadjadj S, Cariou B, Fumeron F, et al. Death, endstage renal disease and renal function decline in patients with diabetic nephropathy in French cohorts of type 1 and type 2 diabetes. Diabetologia. 2016;59:208-16.

21. Saran R, Li Y, Robinson B, et al. US Renal Data System 2014 annual data report: epidemiology of kidney disease in the United States. Am J Kidney Dis. 2015;66(1 Suppl 1):Svii-305.

22. Saran R, Robinson B, Abbott KC, et al. US Renal Data System 2016 annual data report: epidemiology of kidney disease in the United States. Am J Kidney Dis. 2017;69:A7-8.

23. Russ KB, Stevens TM, Singal AK. Acute kidney injury in patients with cirrhosis. J Clin Transl Hepatol. 2015;3:195-204.

24. Alicic RZ, Rooney MT, Tuttle KR. Diabetic kidney disease: challenges, progress, and possibilities. Clin J Am Soc Nephrol. 2017;12:2032-45.

25. Tziomalos K, Athyros VG. Diabetic nephropathy: new risk factors and improvements in diagnosis. Rev Diabet Stud. 2015;12:110-8.

26. Gheith O, Farouk N, Nampoory N, Halim MA, AlOtaibi T. Diabetic kidney disease: world wide difference of prevalence and risk factors. J Nephropharmacol. 2016;5:49-56.

27. Patel SS. Cerebrovascular complications of diabetes: alpha glucosidase inhibitor as potential therapy. Horm Metab Res. 2016;48:8-91.

28. Zhang M, Chen $\mathrm{P}$, Chen $\mathrm{S}$, et al. The association of new inflammatory markers with type 2 diabetes mellitus and macrovascular complications: a preliminary study. Eur Rev Med Pharmacol Sci. 2014;18:1567-72.

29. Chang RE, Lin SP, Aron DC. A pay-for-performance program in Taiwan improved care for some diabetes patients, but doctors may have excluded sicker ones. Health Aff (Millwood). 2012;31:93-102.

30. Cheng SH, Lee TT, Chen CC. A longitudinal examination of a pay-for-performance program for diabetes care: evidence from a natural experiment. Med Care. 2012;50:109-16.

31. Prakash J, Lodha M, Singh SK, Vohra R, Raja R. Diabetic retinopathy is a poor predictor of type of nephropathy in proteinuric type 2 diabetic patients. J Assoc Phys India. 2007;55:412-6.

32. Hung CC, Lin HY, Hwang DY, et al. Diabetic retinopathy and clinical parameters favoring the presence of diabetic nephropathy could predict 
renal outcome in patients with diabetic kidney disease. Sci Rep. 2017;7:1236.

33. Dong Z, Wang Y, Qiu Q, et al. Clinical predictors differentiating non-diabetic renal diseases from diabetic nephropathy in a large population of type 2 diabetes patients. Diabetes Res Clin Pract. 2016;121:112-8.

34. Soleymanian T, Hamid G, Arefi M, et al. Non-diabetic renal disease with or without diabetic nephropathy in type 2 diabetes: clinical predictors and outcome. Ren Fail. 2015;37:572-5.

35. Prakash J. Non-diabetic renal disease (NDRD) in patients with type 2 diabetes mellitus (type $2 \mathrm{DM}$ ). J Assoc Physicians India. 2013;61:194-9.
36. Lin CC, Lai MS, Syu CY, Chang SC, Tseng FY. Accuracy of diabetes diagnosis in health insurance claims data in Taiwan. J Formos Med Assoc. 2005;104:157-63.

37. Chen YC, Yeh HY, Wu JC, Haschler I, Chen TJ, Wetter T. Taiwan's National Health Insurance Research Database: administrative health care database as study object in bibliometrics. Scientometrics. 2011;86:365-80.

38. Lin LY, Warren-Gash C, Smeeth L, Chen PC. Data resource profile: the National Health Insurance Research Database (NHIRD). Epidemiol Health. 2018;40:e2018062. 